
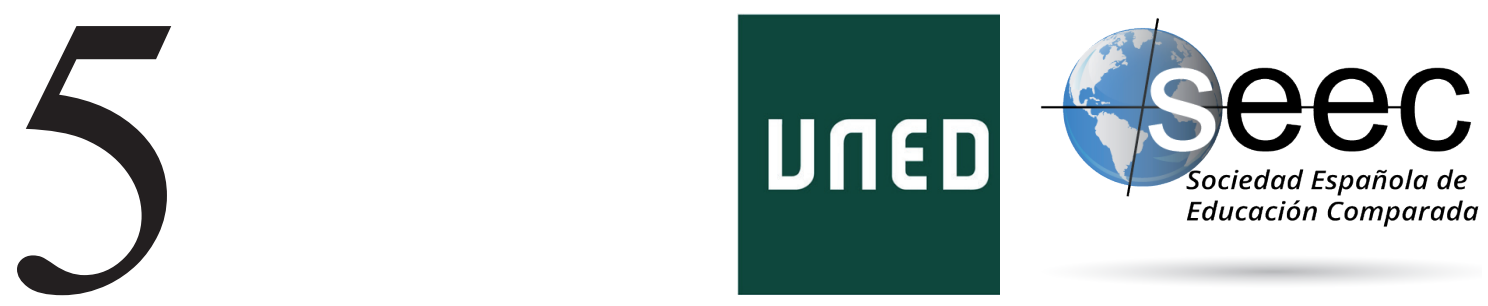

\title{
Equidad estructural en los sistemas educativos europeos
}

Structural equity in European educational systems

\section{Antonia Lozano Díaz*; Juan Sebastián Fernández- Prados $^{* *}$}

DoI: $10.5944 /$ reec.32.2018.22857

Recibido: 7 de octubre de 2018 Aceptado: 28 de diciembre de 2018

\footnotetext{
*Antonia Lozano Díaz: Doctora en Psicología por la Universidad de Almería. Profesora Asociada. Departamento de Educación. Facultad de Ciencias de la Educación. ORCID: https://orcid.org/oooo-00020837-7932. Datos de contacto: E-mail: ald805@ual.es

*Juan Sebastián Fernández-Prados: PT.U. Área de Sociología. Departamento de Historia, Geografía y Humanidades. Facultad de Educación. Universidad de Almería. ORCID: HTTP://orcID.org/oooo-00027419-3998. Datos de contacto: E-mail: jsprados@ual.es
} 


\title{
Resumen
}

La Unión Europea ha apostado por convertirse en una economía competitiva y dinámica sin perder por ello cohesión social. La educación es uno de los pilares en los que se basa para alcanzar dicho objetivo, pero también un elemento crucial desde el que velar por la equidad y la cohesión social. El presente estudio aborda, con un enfoque cualitativo mediante la investigación documental, qué medidas estructurales de equidad se están desarrollando desde los sistemas educativos de 32 países europeos y qué perfiles de equidad pueden establecerse entre los países. El análisis permite mostrar avances en la línea pretendida, pero también el uso de prácticas y políticas educativas incoherentes e incluso contrarias a la equidad y la inclusión social y educativa.

Palabras clave: equidad, educación inclusiva, sistemas educativos

\begin{abstract}
The European Union has opted for a competitive and dynamic economy without losing social cohesion. Education is one of the pillars on which it is based to achieve this objective, but also a crucial element from which to ensure equity and social cohesion. The present study approaches, with a qualitative approach through documentary research, what equity measures are being developed from the educational systems of 32 European countries and what equity profiles can be established between countries. The analysis allows to show progress in the line intended, but also the use of practices and educational policies inconsistent and even contrary to equity and social and educational inclusion.
\end{abstract}

Key Words: equity, inclusive education, educational systems 


\section{Introducción}

La Estrategia de Lisboa marcó como objetivo para la Unión Europea hacer de su economía la economía del conocimiento más competitiva y dinámica del mundo, capaz de un crecimiento económico duradero acompañado por una mejora cuantitativa y cualitativa del empleo y una mayor cohesión social. El alcance de este objetivo supone, entre otros, modernizar el modelo social europeo invirtiendo en recursos humanos y luchando contra la exclusión social. De entre los pilares en los que se basa para lograrlo la Unión Europea (en adelante UE), destaca la educación como eje fundamental desde el que promover la equidad, la cohesión social y la ciudadanía activa. La cohesión social alude a un sentido de identidad compartida, con una distribución equitativa de derechos, oportunidades, riquezas e ingresos y una sociedad civil fuerte (Green, Preston y Sabates, 2003), estos aspectos buscan ser abordados a través de diversas medidas estructurales de equidad en las políticas educativas. Estas actuaciones se enmarcan en un Marco Estratégico para la Educación y la Formación que en principio llegaba hasta 2015, pero que se ha ampliado hasta 2020 (Comisión Europea, 2013). Como consecuencia, los diversos países de la UE, y algunos extracomunitarios, se proponen ajustar su política educativa a fin de encaminar sus sistemas educativos en la línea comentada.

La nueva filosofía resultante alude a la llamada escuela inclusiva que busca integrar la diferencia en el centro educativo de modo abierto a la sociedad para conseguir la plena integración social del alumnado (Serrano, Palomares y Kostich, 2016). La inclusión se considera una dimensión de la equidad junto a la noción de justicia, implica garantizar que las circunstancias personales y sociales no supongan un obstáculo educativo y asegurar un estándar de educación para todos (Echeita, 2013). El modelo de educación inclusiva supone asumir el reconocimiento de la diversidad de alumnado mediante la puesta en funcionamiento de políticas educativas y propuestas que busquen garantizar el éxito para todo el alumnado (Mata y Ballesteros, 2012). Es importante destacar además la evolución del concepto de diversidad que ha pasado de una visión reduccionista, centrada en el problema del alumnado (Navarro-Montaño, 2017), a una visión de inclusividad que supone asumir la diferencia intrínseca de cada persona y responder con una educación para todos (Domínguez et al., 2016).

$\mathrm{El}$ análisis de la equidad de los sistemas educativos es una inquietud que surge con cierta frecuencia como lo demuestran estudios sobre España (Bonal, 2003), África y Centroamérica (Vélaz de Medrano y Rodríguez, 2012), México (Alcántara y Navarrete, 2014), Brasil y Chile (Donoso-Díaz et al., 2016) o a nivel supranacional como el de PISA (Organisation for Economic Co-operation and Development, OECD, 2015) o sobre política educativa de organismos como la UNESCO (Represas, 2014). Es importante reseñar que la equidad en educación alude a la igualdad proporcional de recursos y oportunidades entre los diferentes sectores sociales (Seibold, 2000).

En resumen, el Horizonte 2020 de la Unión Europea establece la apuesta por un crecimiento económico duradero, la mejora del empleo y la cohesión social. La educación se considera uno de los ejes primordiales desde los que promover la equidad, la cohesión social y la ciudadanía activa. Factores como la descentralización de la administración educativa, la consideración de las lenguas minoritarias, las becas y ayudas, la gratuidad de la educación preescolar, la duración de la educación básica, la existencia de un programa educativo común o no, la formación continua del profesorado, la atención a la diversidad y la inversión en educación son analizados en 32 países europeos ( 28 de ellos 
de la Unión Europea). Los resultados obtenidos muestran prácticas educativas no siempre coherentes entre las políticas educativas y las pretensiones políticas relacionadas con la equidad y la cohesión social de la Unión Europea.

\section{Indicadores de equidad}

El logro de la cohesión social mediante la equidad educativa supone valorar e incorporar la diversidad cultural de los diversos colectivos existentes en el contexto. Ello implica garantía y protección desde las instituciones responsables implicadas. Las medidas de equidad educativa por las que se interesa y apuesta la UE resultan coherentes con las diversas aportaciones e investigaciones sobre dicho tópico. Algunas investigaciones relacionadas con dichas medidas de equidad se comentan a continuación:

Descentralización de la administración educativa. El acercamiento al entorno de cada centro educativo o contextualización es un elemento de equidad recurrente en diversas investigaciones como las de Arnáiz y Escarbajal (2012), Pérez et al. (2016), Pérez y Soto (2011) y Pedró (2012). Civís y Longás (2015) Díaz y Civís (2011) defienden la necesidad de crear redes socioeducativas que sitúen a la escuela en el núcleo del entramado para así mejorar la inclusión social y educativa del alumnado.

Lenguas de instrucción. La construcción de una identidad colectiva no excluyente supone tener en cuenta la lengua y cultura de la población inmigrante (Madariaga et al., 2013) así como las lenguas minoritarias o cooficiales de cada país (Vez, 2009). Los valores compartidos por las diversas culturas, valores transculturales, son relevantes educativamente como agentes promotores de cohesión social (Ibáñez-Martín et. al., 2012).

Becas y ayudas. El apoyo a través de becas y ayudas económicas al estudiante y la familia se muestran como un elemento relevante para garantizar la equidad educativa (Berlanga et al., 2018; Marchesi, 2000; Pérez y Sarrate, 2013). Para prevenir el abandono el alumnado más pobre necesita ayuda y protección más allá de la educación gratuita (Abuya, Oketch y Musyoka, 2012).

Gratuidad de la educación preescolar. La escolarización lo más temprana posible dando prioridad a la etapa preescolar es una de las medidas de equidad recomendadas por la OECD (2008) y una decidida apuesta en los sistemas educativos a lo largo del planeta (Sánchez y Mayo, 2014). Pedró (2012), establece que «niveles relativamente altos de matriculación en educación preescolar se asocian con bajos efectos del entorno socioeconómico de la escuela en los resultados de los alumnos» (p. 38). La asistencia a preescolar de niños en desventaja reduce la posibilidad de fracaso escolar y mejora sus posibilidades futuras (Lamy, 2013), compensa estrategias fundamentales no adquiridas en el hogar como un buen nivel lingüístico (Agirdag, Van Avermaet y Van Houtte, 2013) y correlaciona con un alto nivel en lectura y matemáticas a los 15 años con independencia del efecto de la clase social (Agirdag, Yazici y Sierens, 2015).

Duración de la enseñanza obligatoria. La duración de la educación obligatoria y el tiempo de instrucción es un factor importante para el aprendizaje y compensa debilidades en otros ámbitos (Comisión Europea/Eurydice, 2017; Rodríguez, Ríos y Racionero, 2012; Marchesi, 2000).

Existencia de un programa educativo común. El aplazamiento de elecciones académicas tempranas y la existencia de una vía educativa común en la educación obligatoria constituye una medida de equidad recomendada por la OECD (2008), Torres (s.f.) y Domínguez et al. (2016). Según Pedró (2012), la diferenciación curricular temprana de 
los alumnos no produce mejoras en el rendimiento escolar y tiende a asociarse con un aumento en el ámbito de las desigualdades sociales y económicas.

Formación continua. La formación del profesorado ya sea de tipo inicial o continua se considera un elemento clave para el correcto desarrollo de políticas inclusivas (Torres y Castillo, 2016; Blecker y Boakes, 2010). Una mayor cualificación de los docentes se relaciona con una menor influencia de un entorno desfavorable en el rendimiento escolar (Pedró, 2012).

Atención a la diversidad. Según Marchesi (2000) la escolarización del alumnado con necesidades educativas especiales en centros ordinarios son indicadores de equidad hacia este colectivo. Autores como Pedró (2012), encuentran en un análisis de varios países que la segregación de alumnos por capacidades tiene un efecto negativo en su rendimiento. Echeita (2013) y Causa y Chapuis (2010) defienden que el apoyo a este alumnado debe hacerse dentro del grupo ordinario para no perjudicar su aprendizaje y obtener buenos resultados. Atender al alumnado inmigrante supone dotarle de la lengua vehicular pero también potenciar programas de aprendizaje de la lengua materna (Martínez-Usarralde, Fernández-García y Ayala-De la Peña, 2016).

Inversión en educación. La educación debe entenderse como una inversión a largo plazo con gastos usuales que requieren una financiación previsible y sostenible (RonBalsera, Dorsi y Petersen, 2016). La reducción de desigualdades educativas supone prolongar la educación de los más pobres, con las medidas económicas y sociales necesarias, y con la mejora de la calidad de la enseñanza (Marchesi, 2000), para ello el mecanismo de gasto debe adaptar los recursos a las necesidades del contexto (Causa y Chapuis, 2010).

Los anteriormente comentados van a ser los indicadores por considerar dentro de este trabajo, aunque sin dejar de tener en cuenta que «Ninguna selección de indicadores es neutral, sino que refleja la voluntad del investigador de mostrar determinados aspectos de la realidad educativa y social» (Bonal, 2003, p. 61). Por lo tanto, este estudio se centra en factores meramente estructurales sin entrar en otras cuestiones como las diferencias en resultados según clase social, salario según nivel de estudios y similares.

\section{Método}

Los objetivos de la presente investigación son los siguientes: a) conocer qué medidas de promoción de la equidad se están aplicando y de qué modo; b) establecer cuál es el perfil de equidad de los países de la muestra.

El enfoque metodológico a seguir es de índole cualitativo mediante la investigación documental o bibliográfica, uno de los métodos más usuales para acometer un tema de investigación (Pulido et al., 2007). Según Ander-Egg (1993) «si una idea de investigación puede ser formulada de forma tal que el material disponible ya recopilado contenga en si la respuesta a tal pregunta, el uso de ese material es posible» (p. 123). Al mismo tiempo es de carácter descriptivo puesto que pretende establecer si se están abordando o no indicadores de equidad. Para recoger dicha información nos serviremos de la información contenida en la página web de la Red Eurydice en su apartado "National Education Systems". Del total de 38 países que se recogen en tal apartado se seleccionaron únicamente los países que informaban sobre todos los indicadores de equidad que interesan a nuestro estudio, 32 en total. Los países que informan sobre los aspectos comentados de su sistema educativo en Eurydice son los pertenecientes a la Unión Europea: Austria, Bélgica, Bulgaria, Croacia, Chipre, República Checa, Dinamarca, Estonia, Finlandia, Francia, 
Alemania, España, Grecia, Hungría, Irlanda, Italia, Letonia, Lituania, Luxemburgo, Malta, Países Bajos, Polonia, Portugal, Rumanía, Eslovaquia, Eslovenia, Suecia y Reino Unido. A ellos se suman otros países como Islandia, Liechtenstein, Noruega y Suiza.

La Red Eurydice (European Commission, 2015) se encarga de llevar a cabo análisis sobre la organización educativa de los diversos países de Europa. Publica estudios comparativos sobre diversos tópicos e indicadores educativos, así como descripciones de los sistemas educativos nacionales, que se ofrece como recurso online de la Red Eurydice, con una descripción precisa y actualizada de la organización de los sistemas educativos europeos y sus reformas más recientes. Se trata de un documento en línea que los propios países van actualizando con sus datos. Para llevar a cabo la descripción de los diversos sistemas educativos se sirve de una serie de descriptores sobre el contexto social, político y económico del país, la organización general del sistema educativo; la financiación de la educación, las etapas educativas, la formación continua, el personal educativo y profesorado, el apoyo y atención a la diversidad, la movilidad y las reformas en curso. De entre estos descriptores, organizados por capítulos, se han analizado aquellos que recogían indicadores de equidad tal y como se recoge en la Tabla 1.

En resumen, el procedimiento seguido para establecer los criterios de equidad y los niveles dentro los mismos es el siguiente:

- análisis de los diversos capítulos de la web de la Red Eurydice en su apartado "National Education Systems" para ver qué aspectos relacionados con la equidad recogen.

- análisis de cada uno de los indicadores de equidad para establecer categorías dentro de ellos.

Tabla 1

Capítulos de "National Education Systems" que recogen indicadores de equidad

\begin{tabular}{ll}
\multicolumn{1}{c}{$\begin{array}{c}\text { SISTEMAS EDUCATIVOS NACIONALES } \\
\text { (EURYDICE) }\end{array}$} & \multicolumn{1}{c}{ INDICADORES DE EQUIDAD } \\
\hline Cap.1 Contexto político, económico y social & Lenguas de instrucción \\
\hline Cap. 2 Organización y administración SE & $\begin{array}{l}\text { Descentralización de la administración } \\
\text { educativa }\end{array}$ \\
\hline Cap. 3 Financiación de la educación & Becas y ayudas \\
\hline Cap. 4 Educación Infantil & Gratuidad de la educación preescolar \\
\hline Cap. 5 Educación Primaria & Duración de la educación obligatoria \\
Cap. 6 Educación Secundaria & Existencia de un programa común/itinerarios \\
\hline Cap. 9 Profesorado & Formación continua \\
Cap. 12 Apoyo educativo y orientación & Atención a la diversidad \\
\hline
\end{tabular}

Fuente: Elaboración propia. 
Para el criterio de inversión en educación se ha recurrido como fuente a EUROSTAT. Este analiza los datos sobre el gasto del gobierno general en «educación» (de acuerdo con la Clasificación de las Funciones del Gobierno - COFOG). Es parte de un conjunto de informes estadísticos basados en los gastos del gobierno por función en el marco del Sistema Europeo de Cuentas Nacionales (ESA2010).

Se establecen a continuación la descripción de tales indicadores, así como las tipologías hallados en función de su ajuste o no a criterios de equidad:

Descentralización de la administración educativa. La descentralización se refiere básicamente al reparto de responsabilidades educativas a diversos niveles y que por tanto no dependen en exclusiva de las altas instancias, usualmente el Ministerio de Educación. Un ejemplo de este indicador son las Comunidades Autónomas en España y los Lander en Alemania. La tipología hallada fue sencilla: a) administración descentralizada; b) administración centralizada

Lenguas de instrucción. Supone tener en cuenta, o no, otras lenguas para la instrucción. Las tipologías halladas fueron: a) solo una lengua oficial (aunque existan lenguas minoritarias), b) una o más lenguas cooficiales y c) una lengua oficial y lenguas minoritarias (solo para la instrucción).

Becas y ayudas. La tipología de ayudas fue la siguiente: a) bonificación por hijo con independencia de la renta; b) Gratuidad de libros de texto; c) Gratuidad de libros de texto, comida y/o transporte; d) Becas y ayudas según renta.

Gratuidad de la educación preescolar. En este apartado se tiene en cuenta la oferta gratuita de escolarización desde el nacimiento hasta la edad de escolarización obligatoria. Las tipologías halladas fueron: a) E. Preescolar gratis los 2-3 años previos a la educación obligatoria, b) Ningún tramo gratuito.

Duración de la enseñanza obligatoria. Tiempo de escolarización obligatoria que comprende tanto la educación primaria como parte de la secundaria. Tipologías: a) 12 años, b) 11 años, c) 10 años, d) 9 años.

Existencia de un programa común vs itinerarios formativos. Alude al hecho de que todo el alumnado siga un programa educativo común a lo largo de toda la enseñanza obligatoria o existan itinerarios educativos antes de finalizar la misma. Entre los países que establecen itinerarios se han encontrado las siguientes tipologías en función de la edad de comienzo: a) desde los 15 años, b) desde los 14 años, c) desde los 12 años, d) desde los 11 años, f) desde los 10 años.

Formación continua. La formación continua y la actualización del profesorado es una inquietud y necesidad muy contemplada en el total de países. La oferta de formación se ubica en universidades, en centros específicos de formación del profesorado o en otros centros con la cualificación oportuna. La tipología hallada en este caso fue el nivel de obligatoriedad para el profesorado en ejercicio: a) obligación profesional y contractual, b) obligación solo profesional.

Atención a la diversidad. Se refiere a la organización de la respuesta educativa para el alumnado en función de sus particularidades, las tipologías halladas fueron: a) establecimiento de categorías de alumnado con necesidades educativas especiales, b) establecimiento de niveles de apoyo educativo (no de alumnado con necesidades educativas especiales), c) medidas para el alumnado en desventaja sociocultural, d) medidas para el alumnado inmigrante.

Inversión en educación. Alude a la cantidad del PIB que el país destina a educación.

A destacar el hecho de que solo se va a tener en cuenta el período de edad desde el nacimiento hasta el final de la enseñanza obligatoria. 
Como puede observarse se trata de elementos y factores meramente estructurales que proporcionan información sobre la voluntad en política educativa de cada país. Sin embargo, no considera factores como la desigualdad de acceso a la educación según nivel educativo de los padres, las diferencias en resultados según clase social, empleabilidad según nivel de estudios, etc. Se trata de elementos clave en equidad pero que exceden las pretensiones de esta investigación.

\section{Resultados}

A continuación, se comentan los resultados que para cada criterio de equidad arrojan los diversos países según lo comentado en el apartado del método y que se muestran en la Tabla 2.

Tabla 2

Indicadores de equidad en el Sistema Educativo

\begin{tabular}{ll}
\hline \multicolumn{1}{c}{ INDICADORES } & \multicolumn{1}{c}{ PAÍSES (32 en total) } \\
\hline 1. Descentralización de la Administración Educativa \\
\hline a) administración descentralizada & 30 países \\
\hline b) administración centralizada & Chipre y Malta \\
\hline 2. Lenguas de instrucción & \\
\hline a) solo una lengua oficial & $\begin{array}{l}\text { Alemania, Francia, Bulgaria, Islandia, Portugal y República } \\
\text { Checa }\end{array}$ \\
\hline b) una o más lenguas cooficiales & $\begin{array}{l}\text { Austria, Bélgica, Chipre, Eslovenia, España, Estonia Finlandia, } \\
\text { Irlanda, Liechtenstein, Luxemburgo, Malta, Noruega, Reino } \\
\text { Unido y Suiza. }\end{array}$ \\
\hline c) una lengua oficial + lenguas & Croacia, Eslovaquia, Grecia, Hungría, Italia, Letonia, Lituania, \\
minoritarias (solo para instrucción) & Países Bajos, Polonia, Rumanía y Suecia \\
\hline
\end{tabular}

\section{Becas y ayudas}

a) bonificación por hijo/a con independencia de la renta.

b) gratuidad de los libros de texto
Austria, Bélgica (comunidad Flamenca), Chipre, Estonia, Hungría, Letonia, Luxemburgo, Reino Unido, Suecia y Suiza España, Francia, Grecia, Luxemburgo, Malta, Reino Unido, Rumanía y Suiza

Austria, Bélgica (Comunidad Alemana), Dinamarca, Estonia, Finlandia, Polonia y Suecia

c) gratis libros de texto + comida y/o transporte

Todos los países excepto Dinamarca y Suecia

d) Becas y ayudas según renta

4. Gratuidad de la educación preescolar
a) al menos 2 ó 3 años de escolaridad gratis
30 países

b) ningún año gratis Dinamarca y Noruega

(Continua en la siguiente página) 
INDICADORES

PAÍSES (32 en total)

\section{Duración de la enseñanza obligatoria}

\begin{tabular}{ll}
\hline a) 12 años & Bélgica, Países Bajos y Portugal \\
\hline b) 11 años & Malta, Reino Unido y Rumanía \\
\hline \multirow{2}{*}{ c) 10 años } & Alemania, Dinamarca, Francia, España, Hungría, Islandia, \\
& Irlanda, Italia, Luxemburgo y Noruega \\
\hline & Austria, Bulgaria, Croacia, Chipre, Eslovaquia, Eslovenia, \\
d) 9 años & Estonia, Finlandia, Grecia, Letonia, Liechtenstein, Lituania, \\
& Polonia, República Checa, Suecia y Suiza \\
\hline
\end{tabular}

6. Existencia de itinerarios

\begin{tabular}{ll}
\hline a) desde los 15 años & Francia, Irlanda, Portugal, Rumanía \\
\hline b) desde los 14 años & Bélgica, Bulgaria, España, Hungría y Italia \\
\hline c) desde los 12 años & Alemania, Luxemburgo y Países Bajos \\
\hline d) desde los 11 años & Eslovaquia y Lituania \\
\hline e) desde los 10 años & Austria \\
\hline
\end{tabular}

7. Formación continua

a) obligación profesional y contractual

) solo obligación profesional

Alemania, Austria, Bélgica (excepto comunidad Flamenca), Croacia, Chipre, Eslovenia, Finlandia, Grecia, Hungría, Islandia, Italia, Letonia, Luxemburgo, Malta, Rumanía, Reino Unido (Inglaterra y Gales), República Checa y Suiza

Bulgaria, Dinamarca, Eslovaquia, España, Estonia, Francia, Irlanda, Liechtenstein, Lituania, Malta, Noruega, Países Bajos, Portugal y Suecia

\section{Atención a la diversidad}

\begin{tabular}{ll}
\hline a) niveles de apoyo educativo & Alemania, Finlandia, Noruega y Suecia \\
\hline $\begin{array}{l}\text { b) categorización de alumnado con } \\
\text { necesidades educativas }\end{array}$ & Todos (salvo los 4 anteriores) \\
\hline d) medidas para alumnado en desventaja & Todos \\
\hline e) medidas para alumnado inmigrante & Todos (excepto Chipre) \\
\hline
\end{tabular}

Fuente: Elaboración propia.

Con relación a la Descentralización de la Administración Educativa, el proceso de gestión y administración se desarrolla a lo largo de un espectro que va desde aquellos países que permiten a nivel regional tanto la gestión como la regulación normativa, hasta aquellos que permiten solo aspectos básicos de gestión como los criterios de admisión en centros y similares. Los países con una administración totalmente centralizada son Malta y Chipre. La organización administrativa también está muy relacionada con la tradición histórica de cada país como es el caso de los Lander en Alemania, las Comunidades Autónomas en España, los Cantones en Suiza o las Regiones en Bélgica y Reino Unido. Son estos países los que mayor nivel de descentralización presentan. 
En relación a las Lenguas de Instrucción en todos los países de la muestra hay más de una lengua hablada en el país. Los países con una sola lengua de instrucción, a pesar de reconocer otras lenguas minoritarias, son Alemania, Francia, Portugal, Bulgaria, República Checa e Islandia. Como puede observarse en la Tabla 2 hasta 14 países reconocen otras lenguas cooficiales, es decir lenguas al mismo nivel administrativo que la lengua oficial de todo el estado, y que por tanto son también lenguas de instrucción. Otros 12 países ofrecen la posibilidad de recibir instrucción en lenguas minoritarias (solo tienen estatus oficial para la instrucción).

En el apartado de Becas y ayudas son 10 los países que bonifican el nacimiento de hijo/a al margen de la renta familiar. Como puede observarse en la Tabla 2, todos los países ofrecen becas y ayudas de diverso tipo en función de la renta familiar a excepción de Suecia y Dinamarca porque también son los únicos que ofrecen, totalmente gratis, los libros de texto, comida y transporte escolar. Sin embargo, destacan aquí dos países en particular, Austria, Estonia y parte de Bélgica, ya que son los que más amplitud de ayudas en la educación obligatoria conceden: bonificación por hijo nacido, ayudas según renta, así como gratuidad de libros de texto, transporte y/o comida. A destacar el hecho de que, de los 32 países, un total de 14 ofrecen solamente becas y ayudas en función de la renta familiar.

En lo referente a la Gratuidad de la educación preescolar, en la Tabla 2 se muestra como excepto Dinamarca y Noruega todos los países ofertan de modo totalmente gratuito una parte de la educación preescolar. Usualmente suelen los 2-3 años previos al comienzo de la etapa de primaria. Ninguno de los 32 países de la muestra considera la gratuidad desde los o años.

La Duración de la enseñanza obligatoria recoge también amplias diferencias entre países tal y como se puede ver en la Tabla 2. El período de duración más corto para la enseñanza obligatoria es de 9 años, y se desarrolla en la mitad de los países de la muestra. El siguiente período es de 10 años de educación obligatoria y se lleva a cabo en 10 países. La enseñanza obligatoria de 11 años se considera en 3 países de la muestra. La educación obligatoria de mayor duración es la que abarca un total de 12 años, los países que han adoptado este período son Portugal, Países Bajos y Bélgica. A destacar el hecho de que entre los países de mayor y menor duración de la enseñanza obligatoria hay una diferencia de 3 años de escolaridad.

En relación con el indicador Programa común vs itinerarios formativos frecuentemente se dan dos situaciones. La situación más común es aquella en la que todo el alumnado sigue el mismo programa básico de asignaturas. La otra situación es aquella en la que comienza una división de la secundaria en al menos dos itinerarios: un itinerario encaminado a proseguir estudios superiores académicos y un itinerario profesionalizador encaminado hacia el mundo laboral, donde cada vez es más importante formarse en sus riesgos (Sánchez, 2016). Como puede observarse en la Tabla 2 de los 32 países de la muestra casi la mitad, 15 exactamente, dividen en alguna forma de itinerario la educación secundaria en su tramo obligatorio. Algunos países como Austria, Lituania, Eslovaquia, Alemania, Luxemburgo y Países Bajos llevan a cabo una división en itinerarios desde el comienzo de la educación secundaria.

La Formación continua del profesorado y su actualización es una inquietud y necesidad muy contemplada en el total de países. La oferta de formación se ubica en universidades, en centros específicos de formación del profesorado o en otros centros con la cualificación oportuna. Más de la mitad, 18 países, de la muestra establece de modo 
obligatorio la formación continua del profesorado: Austria, Bélgica (excepto comunidad Flamenca), Croacia, Chipre, República Checa, Finlandia, Alemania, Grecia, Hungría, Islandia, Italia, Letonia, Luxemburgo, Malta, Rumanía, Eslovenia, Suiza y Reino Unido (Inglaterra y Gales). En el resto de los países la formación continua se recoge como una obligación profesional pero no es una obligación contractual.

Con relación al indicador Atención a la diversidad, en la Tabla 2 se observa como todos los países llevan a cabo una categorización del alumnado con necesidades específicas de apoyo educativo, es decir el foco del problema se sitúa en las dificultades del alumnado en lugar de hacerlo en las dificultades o demandas al sistema para que les de una respuesta educativa acorde con sus particularidades. La excepción son Finlandia, Alemania, Suecia y Noruega que establecen niveles de apoyo educativo en lugar de categorías de necesidades. De este modo el foco de atención del problema se traslada sobre la acción educativa en lugar de hacerlo sobre el alumnado. Asimismo, el total de la muestra de países desarrollan medidas educativas de atención a población inmigrante y a alumnado en situación de desventaja sociocultural. Chipre es el único país que no manifiesta tener medidas específicas para el alumnado inmigrante.

Para analizar la Inversión en educación se ha acudido a los datos de Eurostat (2016). Tal y como puede observarse en el Gráfico 1, la media de PIB en Europa de inversión en educación es del 4.7\%. De los 32 países de la muestra, 10 países invierten menos del 4.7 \% de su PIB en educación. El país que más recursos financieros destina a educación es Dinamarca, el 6.9 \% de su PIB. Este porcentaje supone más del doble de la inversión en educación que lleva a cabo el gobierno del país con menor esfuerzo financiero en educación, Irlanda. Este país dedica poco más del 3 \% de su PIB a la educación. Los países que mayor PIB dedican a la educación en su país son, por orden de mayor a menor, Dinamarca, Suecia, Bélgica, Finlandia y Chipre.

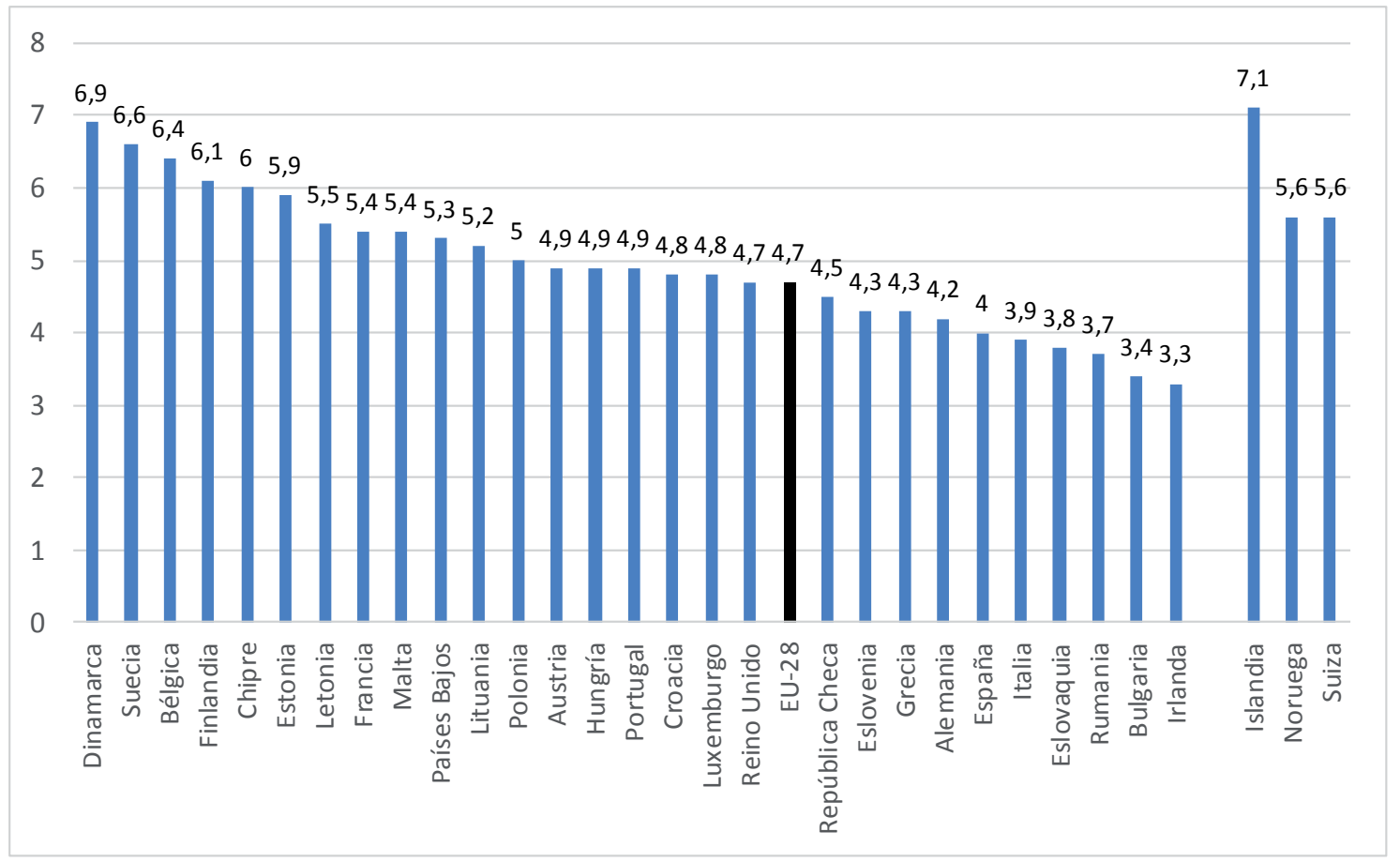

Gráfico 1. Gastos públicos general de los gobiernos en educación en 2016 (\% del PIB). Fuente: Eurostat. 
Finalmente, se ha construido un índice de equidad estructural de los sistemas educativos europeos (IEESEE) en base a los nueve indicadores empleados, más con la intención de presentar una síntesis sinóptica que la de mostrar un instrumento definitivo. Es decir, cabe aún perfeccionar y precisar esta herramienta comparativa de los sistemas educativos desde el punto de vista de la equidad, no solo añadiendo otros indicadores como los que se señalan en estudios internacionales sobre educación (OECD, 2018b) o las encuestas internacionales sobre opinión pública (Fernández, Cuenca y González, 2018), si no también homogeneizando criterios espaciales (regiones dentro de los países) dimensionales (dado que algunos indicadores contemplan varios subindicadores), ponderación (se ha puntuados todos por igual), etc. En cualquier caso, valga como primera aproximación los resultados presentados en la tabla 3, donde aparecen en lugar destacado por orden países como Bélgica, Finlandia, Austria, Reino Unido, Luxemburgo, Malta, Estonia, Portugal y Suiza.

Tabla 3

Índice de Equidad Estructural de los Sistemas Educativos Europeos (IEESEE)

\begin{tabular}{|c|c|c|c|c|c|c|c|c|c|c|}
\hline & 1. & 2. & 3. & 4. & 5. & 6. & 7. & 8. & 9. & Total \\
\hline Alemania & 1 & 0 & 0 & 1 & $\mathrm{O}$ & 0 & 1 & 1 & 0 & 4 \\
\hline Austria & 1 & $\mathbf{1}$ & $\mathbf{1}$ & $\mathbf{1}$ & $\mathbf{0}$ & $\mathbf{0}$ & 1 & $\mathbf{0}$ & $\mathbf{1}$ & 6 \\
\hline Bélgica & $\mathbf{1}$ & $\mathbf{1}$ & $\mathbf{1}$ & $\mathbf{1}$ & $\mathbf{1}$ & $\mathbf{1}$ & $\mathbf{1}$ & $\mathbf{0}$ & $\mathbf{1}$ & 8 \\
\hline Bulgaria & 1 & $\mathrm{O}$ & 0 & 1 & $\mathrm{O}$ & 1 & 0 & $\mathrm{O}$ & $\mathrm{O}$ & 3 \\
\hline Chipre & $\mathrm{O}$ & 1 & $\mathrm{O}$ & 1 & $\mathrm{O}$ & 0 & 1 & $\mathrm{O}$ & 1 & 4 \\
\hline Croacia & 1 & $\mathrm{O}$ & $\mathrm{O}$ & 1 & $\mathrm{O}$ & 0 & 1 & $\mathrm{O}$ & 1 & 4 \\
\hline Dinamarca & 1 & 0 & 1 & 1 & $\mathrm{O}$ & $\mathrm{O}$ & $\mathrm{O}$ & $\mathrm{O}$ & 1 & 4 \\
\hline Eslovaquia & 1 & 1 & $\mathrm{O}$ & 1 & $\mathrm{O}$ & $\mathrm{O}$ & $\mathrm{O}$ & $\mathrm{O}$ & $\mathrm{O}$ & 3 \\
\hline Eslovenia & 1 & $\mathrm{O}$ & $\mathrm{O}$ & 1 & $\mathrm{O}$ & $\mathrm{O}$ & 1 & $\mathrm{O}$ & $\mathrm{O}$ & 3 \\
\hline España & 1 & 1 & $\mathrm{O}$ & 1 & $\mathrm{O}$ & 1 & $\mathrm{O}$ & $\mathrm{O}$ & $\mathrm{O}$ & 4 \\
\hline Estonia & $\mathbf{1}$ & $\mathbf{1}$ & $\mathbf{1}$ & $\mathbf{1}$ & $\mathbf{O}$ & $\mathbf{0}$ & $\mathbf{o}$ & $\mathbf{o}$ & $\mathbf{1}$ & 5 \\
\hline Finlandia & $\mathbf{1}$ & $\mathbf{1}$ & $\mathbf{1}$ & $\mathbf{1}$ & $\mathbf{O}$ & $\mathbf{0}$ & $\mathbf{1}$ & $\mathbf{1}$ & $\mathbf{1}$ & 7 \\
\hline Francia & 1 & $\mathrm{O}$ & $\mathrm{O}$ & 1 & $\mathrm{O}$ & 1 & $\mathrm{O}$ & $\mathrm{O}$ & 1 & 4 \\
\hline Grecia & 1 & $\mathrm{O}$ & $\mathrm{O}$ & 1 & $\mathrm{O}$ & $\mathrm{O}$ & 1 & $\mathrm{O}$ & $\mathrm{O}$ & 3 \\
\hline Hungría & $\mathbf{1}$ & $\mathbf{o}$ & $\mathbf{o}$ & $\mathbf{1}$ & $\mathbf{o}$ & $\mathbf{1}$ & $\mathbf{1}$ & $\mathbf{o}$ & $\mathbf{1}$ & 5 \\
\hline Irlanda & 1 & 1 & $\mathrm{O}$ & 1 & $\mathrm{O}$ & 1 & $\mathrm{O}$ & $\mathrm{O}$ & $\mathrm{O}$ & 4 \\
\hline Islandia & 1 & $\mathrm{O}$ & $\mathrm{O}$ & 1 & $\mathrm{O}$ & $\mathrm{O}$ & 1 & $\mathrm{O}$ & 1 & 4 \\
\hline Italia & 1 & $\mathrm{O}$ & $\mathrm{O}$ & 1 & $\mathrm{O}$ & 1 & 1 & $\mathrm{O}$ & $\mathrm{O}$ & 4 \\
\hline Letonia & 1 & $\mathrm{O}$ & $\mathrm{O}$ & 1 & $\mathrm{O}$ & $\mathrm{O}$ & 1 & $\mathrm{O}$ & 1 & 4 \\
\hline Lituania & 1 & $\mathrm{O}$ & $\mathrm{O}$ & 1 & $\mathrm{O}$ & $\mathrm{O}$ & $\mathrm{O}$ & $\mathrm{O}$ & 1 & 3 \\
\hline Luxemburgo & $\mathbf{1}$ & $\mathbf{1}$ & $\mathbf{o}$ & $\mathbf{1}$ & $\mathbf{o}$ & $\mathbf{o}$ & $\mathbf{1}$ & $\mathbf{o}$ & $\mathbf{1}$ & 5 \\
\hline Malta & $\mathbf{0}$ & $\mathbf{1}$ & $\mathbf{0}$ & $\mathbf{1}$ & $\mathbf{1}$ & $\mathbf{0}$ & $\mathbf{1}$ & $\mathbf{o}$ & $\mathbf{1}$ & 5 \\
\hline Noruega & 1 & 1 & $\mathrm{O}$ & 0 & $\mathrm{O}$ & $\mathrm{O}$ & $\mathrm{O}$ & 1 & 1 & 4 \\
\hline Países Bajos & 1 & $\mathrm{O}$ & $\mathrm{O}$ & 1 & 1 & $\mathrm{O}$ & $\mathrm{O}$ & $\mathrm{O}$ & 1 & 4 \\
\hline Polonia & 1 & $\mathrm{O}$ & 1 & 1 & $\mathrm{O}$ & $\mathrm{O}$ & $\mathrm{O}$ & $\mathrm{O}$ & 1 & 4 \\
\hline Portugal & $\mathbf{1}$ & $\mathbf{O}$ & $\mathbf{0}$ & $\mathbf{1}$ & $\mathbf{1}$ & $\mathbf{1}$ & $\mathbf{o}$ & $\mathbf{o}$ & $\mathbf{1}$ & 5 \\
\hline Reino Unido & $\mathbf{1}$ & $\mathbf{1}$ & $\mathbf{0}$ & $\mathbf{1}$ & $\mathbf{1}$ & $\mathbf{O}$ & $\mathbf{1}$ & $\mathbf{O}$ & $\mathbf{1}$ & 6 \\
\hline República Checa & 1 & $\mathrm{O}$ & $\mathrm{O}$ & 1 & $\mathrm{O}$ & $\mathrm{O}$ & 1 & $\mathrm{O}$ & $\mathrm{O}$ & 3 \\
\hline Rumania & 1 & $\mathrm{O}$ & $\mathrm{O}$ & 1 & 1 & 1 & $\mathrm{O}$ & $\mathrm{O}$ & 0 & 4 \\
\hline Suecia & 1 & $\mathrm{O}$ & 1 & O & $\mathrm{O}$ & $\mathrm{O}$ & $\mathrm{O}$ & 1 & 1 & 4 \\
\hline Suiza & 1 & 1 & $\mathbf{0}$ & $\mathbf{1}$ & $\mathbf{0}$ & $\mathbf{0}$ & $\mathbf{1}$ & $\mathbf{O}$ & 1 & 5 \\
\hline
\end{tabular}

Fuente: Elaboración propia. 
Nota explicativa de los indicadores: 1. Descentralización de la administración educativa; 2. Lenguas de instrucción (una o más lenguas cooficiales); 3. Becas y ayudas (gratis libros de texto + comida y/o transporte); 4. Gratuidad de la educación preescolar; 5. Duración de la enseñanza obligatoria ( $>10$ años); 6. Existencia de un programa educativo común ( $<14$ años); 7. Formación continua (obligación profesional y contractual); 8. Atención a la diversidad (con niveles de apoyo educativo); 9. Inversión en educación ( $>$ Media UE= 4,7 \% PIB).

\section{Discusión y conclusiones}

El primer objetivo planteado en este estudio es conocer de qué modo se están aplicando las diversas medidas de promoción de la equidad. La medida en la que más consenso hay en su aplicación es en la descentralización de la administración educativa, los dos únicos países que no la llevan a cabo son muy pequeños por lo que su influencia no tiene especial relevancia. La consideración educativa de las lenguas minoritarias está bastante extendida por lo que llama la atención que dos de los países más importantes de la UE, Francia y Alemania, solventen la cuestión no admitiendo ninguna otra lengua de instrucción excepto la lengua oficial. Sobre todo, si se tiene en cuenta el rico acervo lingüístico de ambos estados. Un aspecto fundamental para reducir las desigualdades sociales estriba en garantizar la cobertura de gastos educativos básicos (Duru-Bellat, 2010), yendo más allá de la educación gratuita (Abuya, 2012). Los dos países que más coberturas económicas de todo tipo ofrecen son Austria y Estonia, seguidos por Suecia y Dinamarca. Un elemento de consenso en los diversos estudios internacionales está en la importancia de la educación preescolar en la evolución educativa posterior y en la cohesión social derivada de ello. A pesar de ello ningún país la oferta de modo gratuito en su totalidad, de hecho, dos países no ofrecen ningún tramo gratuito. Esto supone trasladar a las familias la decisión de ofrecer este tipo de atención educativa a sus hijos, siendo bastante más probable que sean las familias pudientes las que opten por ello (Agirdag et al. 2015).

La duración de la enseñanza obligatoria es otro elemento compensador de desigualdades, la prolongación del tiempo de instrucción es necesario para el logro de las competencias básicas (Rodríguez, Ríos y Racionero, 2012). La mitad de los países estudiados mantienen a su alumnado en el sistema educativo 9 años, es decir 3 años menos que los países que más prolongan la enseñanza obligatoria. La existencia de itinerarios es uno de los aspectos más desaconsejados para favorecer la igualdad educativa ya que viene a ratificar las dificultades de origen del alumnado más pobre reproduciendo la diferencia de clase en el contexto escolar (Duru-Bellat, 2010; Madrid, 2007; Pedró, 2012; Prats, 2010). La convivencia cotidiana en un grupo escolar segregado refuerza tanto los valores como las dificultades del grupo social de origen. A pesar de ello casi la mitad de los países, 15, mantienen una educación obligatoria segregada por itinerarios. De acuerdo con Prats (2010), el tema de los itinerarios en secundaria remite a un debate ideológico sobre el papel que debe jugar la formación profesional en cada momento.

Considerando la atención a la diversidad, son numerosos los estudios que demuestran lo ventajoso y posible de la inclusión de capacidades y diversidades en la misma aula (Rodríguez et al. 2012; Prats, 2010; Craven, Morin, Tracey, Parker y Zhong, 2015), cuidando de no llevar a cabo grupos segregados en grupos ordinarios (Echeita, 2013) para lo cual es fundamental formar al profesorado (Engelbrecht, Savolainen, Nel, Koskela y Okkolin, 2017). Solo en 4 de los 32 sistemas educativos analizados se parte de diseñar currículos y entornos de aprendizaje adaptados a las diferencias individuales en lugar 
de partir de la categoría del déficit del alumnado, es decir la respuesta educativa se va ajustando a las particularidades del alumnado sin necesidad de una categorización previa de sus necesidades educativas. La idea subyacente estriba en partir de que todo el alumnado es igual de diferente. La cuestión del gasto educativo supone una cuestión controvertida puesto que la relación entre resultados educativos e inversión educativa es ambigua, según Pedró (2012), la diferencia estriba en diseñar una política de gasto educativo finalista y centrada en las necesidades de los colectivos más desfavorecidos para reducir desigualdades. Ron-Balsera et al. (2016), aconsejan un 6 \% del PIB en educación para el caso de los países más pobres, en este estudio el referente es el $4.7 \%$ de inversión en la UE situándose por debajo de dicho porcentaje países relativamente pobres como Rumanía, Bulgaria, Croacia y Eslovaquia. A destacar el notable esfuerzo de países como Estonia, Letonia y Portugal con un bajo PIB per cápita pero que invierten cerca del $5 \%$ o más de su PIB en educación.

El segundo objetivo de esta investigación fue establecer el perfil de equidad de los países de la muestra. Para ello se tendrá en cuenta el mayor o menor acercamiento o ajuste de cada país a los diversos niveles promotores de equidad propuestos. El perfil de países con un mayor nivel de equidad es aquel que se caracteriza principalmente por desarrollar una amplia cobertura del gasto educativo de las familias mediante becas y ayudas; permitir otras lenguas de instrucción; parte de la educación preescolar gratuita; programa común para todo el alumnado en la etapa obligatoria; inversión en educación superior al $4.7 \%$ del PIB del país; administración descentralizada y establecer de modo obligatorio la formación continua del profesorado. Este grupo de países estaría encabezado por Finlandia, seguido en orden por Suecia, Reino Unido, Malta, Chipre, Estonia, Letonia, Suiza, Polonia, Islandia y Dinamarca. Dos de estos países, Finlandia y Suecia, atienden a la diversidad del alumnado enfocándose en la respuesta educativa en lugar de hacerlo sobre el déficit del alumnado, también establecen solo 9 años de escolaridad obligatoria. En el otro extremo y con una menor equidad estaría aquellos países que caracterizan principalmente por establecer itinerarios educativos; política de becas y ayudas solo según renta; inversión en educación por debajo del $4.7 \%$ del PIB y corta duración de la educación obligatoria. Este grupo estaría compuesto principalmente por Bulgaria, Eslovaquia, Irlanda y Alemania.

A pesar de que la UE no tiene potestad sobre la regulación educativa de cada país, y solo puede aconsejar y establecer una hoja de ruta orientativa, está claro que estamos ante una Europa a diversas velocidades con países que no terminan de asumir que las desigualdades participan en la construcción de una sociedad más o menos cohesiva (Duru-Bellat, 2010). La UE busca posicionarse en lo que Sánchez y Manzanares (2014), denominan tercera tendencia en equidad educativa «como inclusión educativa, con énfasis en la redistribución de los recursos educativos, generación de situaciones de aprendizaje ajustadas a los ritmos y expectativas de aprendizaje desde un enfoque inclusivo» (p. 24). Este estudio descriptivo muestra claramente países que no parecen confiar en el tándem calidad y equidad y no optan por este enfoque educativo por la justicia social y la cohesión (Kyriakides, Creemers y Charalambous, 2018). La evidencia sin embargo señala cómo desde la política educativa y los centros escolares es posible desarrollar un entorno de atención y posibilidades para todos, sin que ello suponga dejar de educar para la competencia profesional, el espíritu crítico y la responsabilidad. Dado que se trata de un estudio sobre la equidad estructural de los sistemas educativos se hace necesario completar este análisis con estudios posteriores que tengan en cuenta cuestiones como 
los resultados académicos y nivel socioeconómico de las familias, acceso a la educación según procedencia social y geográfica, nivel de ingresos según nivel educativo alcanzado, influencia de la clase social a igual nivel educativo, etc. y que permitirán trazar un perfil más definido de los diversos elementos de equidad educativa. A pesar de los esfuerzos en equidad desde los aspectos estructurales, en la actualidad el origen social sigue siendo el principal determinante en los resultados educativos y sociales según el último informe de 2018 de la OCDE (OECD, 2018a).

\section{Referencias}

Abuya, B., Oketch, M. y Musyoka, P. (2012). Why do pupils dropout when education is "free"? Explaining school dropout among urban poor in Nairobi. Compare: A Journal of Comparative and International Education, 43(6), pp. 740-762. https:// doi.org/10.1080/03057925.2012.707458

Agirdag, O., Van Avermaet, P. y Van Houtte, M. (2013). School Segregation and Math Achievement: A Mixed-Method Study on the Role of Self-Fulfilling Prophecies. Teachers College Record, 115(3), pp. 1-10.

Agirdag, O., Yazici, Z. y Sierens, S. (2015). Trends in preschool enrolment in Turkey: inequal acces and differential consequences. Comparative Education, 51(4), pp. 537-554. https://doi.org/10.1080/03050068.2015.1081796

Alcántara, A. y Navarrete, Z. (2014). Inclusión, equidad y cohesión social en la política de la educación superior en México. Revista Mexicana de Investigación Educativa, 18(6o), pp. 213-239.

Ander-Egg, E. (1993). Métodos de investigación social. Buenos Aires: Magisterio del Río de la Plata

Arnáiz, P. y Escarbajal, A. (2012). Reflexiones sobre cultura, identidad y racismo desde una mirada pedagógica. Teoría de la Educación, 24(2), pp. 83-106

Becker, N. S. y Boakes, N. J. (2010). Creating a learning environment for all children: are teachers able and willing? International Journal of Inclusive Education, 14(5), pp. 435-447. https://doi.org/10.1080/13603110802504937

Berlanga, V., Figuera, M. P. y Pons, E. (2018). Modelo predictivo de persistencia universitaria: alumnado con beca salario. Educación XXI, 21(1), 209-230. https:// doi.org/10.5944/educxx1.20193

Bonal, X. (2003). Una evaluación de la equidad del sistema educativo español. Revista de Educación. 330, pp. 59-82.

Causa, O. y Chapuis, C. (2010). Equity in Student Achievement Across OECD Countries: An Investigation of the Role of Policies. OECD Journal: Economic Studies 1, pp. 1-50.

Civís, M. y Longás, J. (2015). La colaboración interinstitucional como respuesta al desafío de la inclusión socioeducativa. Análisis de 4 experiencias de trabajo en red a nivel local en Cataluña. Educación XXI, 18(1), pp. 213-236. https://doi.org/10.5944/ educxx1.18.1.12318 
Comisión Europea (2013). Comprender las políticas de la Unión Europea. Europa 2020: la estrategia europea de crecimiento. Bruselas: Comisión Europea.

Comisión Europea/EACEA/Eurydice (2017). Análisis comparativo del tiempo de instrucción anual recomendado en la educación obligatoria a tiempo completo en Europa 2016/17. Eurydice-Datos y cifras. Luxemburgo: Oficina de Publicaciones de la UE.

Craven, R. G., Morin, A. J. S., Tracey, D., Parker, P. D., \& Zhong, H. F. (Eds.). (2015). Inclusive education for students with intellectual disabilities. Inclusive education for students with intellectual disabilities. Charlotte, North Carolina: Information Age Publishing.

Díaz, J. y Civís, M. (2011). Redes Socioeducativas promotoras de capital social en la comunidad: un marco teórico de referencia. Cultura y Educación, 23(3), pp. 415429. https://doi.org/10.1174/113564011797330270

Domínguez, J., López, A. y Vázquez, E. (2016). Atención a la diversidad en Educación Secundaria Obligatoria: análisis desde la inspección educativa. Aula Abierta, 44, pp. 70-76. https://doi.org/10.1016/j.aula.2016.03.002

Donoso-Díaz, S., De Souza, A. R. y Barbosa, A. (2016). Desafíos políticos clave para los sistemas escolares de Brasil y Chile. Revista Española de Educación Comparada, 27, pp. 73-96. https://doi.org/10.5944/reec.27.2016.16429

Duru-Bellat, M. (2010). La desigualdad en Europa: una cuestión de actualidad. Revista Española de Educación Comparada, 16, pp. 105-130.

Echeita, G. (2013). Inclusión y exclusión educativa. De nuevo «Voz y quebranto». Revista Iberoamericana sobre Calidad, Eficacia y Cambio en Educación, 11(2), pp. 99-108.

Engelbrecht, P., Savolainen, H., Nel, M., Koskela, T. y Okkolin, M. (2017). Making meaning of inclusive education: classroom practices in Finnish and South African classrooms. Compare: A Journal of Comparative and International Education, 47(5), pp. 684-702. https://doi.org/10.1080/03057925.2016.1266927

European Commission (2015). Countries-Eurydice. Disponible: en: https://eacea. ec.europa.eu/national-policies/eurydice/national-description_en

Felouzis, G. y Charmillot, S. (2012). School tracking and educational inequality: a comparison of 12 education systems in Switzerland. Comparative Education, 49(2), pp. 181-205. https://doi.org/10.1080/03050068.2012.706032

Fernández-Prados, J. S., Cuenca-Piqueras, C., y González-Moreno, M. J. (2018). International public opinion surveys and public policy in Southern European democracies Southern European democracies. Journal of International and Comparative Social Policy, http://doi.org/10.1080/21699763.2018.1535997

Green, A.; Preston, J. y Sabates, R. (2003). Education, Equality and Social Cohesion: A distributional approach. Compare: A Journal of Comparative and International Education, 33(4), pp. 453-470. https://doi.org/10.1080/0305792032000127757 
Ibáñez-Martín, J. A., Fuentes, J. L. y Barrio, J. M. (2012). Competencias sociales e inmigración desde una perspectiva intercultural. Educación XXI, 15(2), pp.41-72. https://doi.org/10.5944/educxx1.15.2.126

Kyriakides, L., Creemers, B., \& Charalambous, E. (2018). Equity and quality dimensions in educational efectiveness. New York: Springer.

Lamy, C. E. (2013). How Preschool fights Poverty. Educational Leadership, 7o(8), pp. $32-36$

Madariaga, J. M., Huguet, A. y Lapresta, C. (2013). Actitud presión social y educación inclusiva en aulas con diversidad lingüística y cultural. Educación XXI, 16(1), pp. 305-328. https://doi.org/10.5944/educxx1.16.1.728

Madrid, J. M. (2007). La política educativa de la Unión Europea al servicio del desarrollo económico con cohesión social. Revista Española de Educación Comparada, 13, pp. 253-284.

Marchesi, A. (2000). Un sistema de indicadores de desigualdad educativa. Revista Iberoamericana de Educación, 23, pp. 135-163.

Martínez-Usarralde, M. J., Fernández-García, C. M. y Ayala-De la Peña, A. (2016). «Yo acojo, tú agrupas, ella compensa». Análisis comparado de las políticas de integración del alumnado inmigrante en tres Comunidades Autónomas. Revista Complutense de Educación, 27(3), pp. 1103-1118. http://dx.doi.org/10.5209/rev_ RCED.2016.v27.n3.47547

Mata, P. y Ballesteros, B. (2017). Diversidad cultural, eficacia escolar y mejora de la escuela: encuentros y desencuentros. Revista de Educación, 358, pp. 17-37.

Navarro-Montaño, M. J. (2007). Mejorar la calidad de la educación inclusiva: propuesta de indicadores. Revista de Pedagogía, 38(102), pp. 122-143.

OECD (2008). Ten Steps to Equity in Education. Policy Brief. [Documento en línea] Disponible: http://www.oecd.org/education/school/39989494.pdf [Consulta: 2018, enero 5].

OECD (2017). PISA 2015 Results (Volume I): Excellence and Equity in Education, PISA. Paris: OECD Publishing.

OECD (2018a). Education at a Glance 2018: OECD Indicators. Paris: OECD Publishing. https://doi.org/10.1787/eag-2018-en

OECD (2018b). Equity in Education: Breaking Down Barriers to Social Mobility, PISA. Paris: OECD Publishing. https://doi.org/10.1787/9789264073234-en

Pedró, F. (2012). Políticas públicas sobre apoyo y refuerzo educativo: evidencias internacionales. Revista de Educación, $n .^{\circ}$ extraordinario, pp. 22-45. https://doi. org/10.4438/1988-592X-RE-2012-EXT-205

Pérez, A. I. y Soto, E. (2011). Luces y sombras de PISA. Sentido educativo de las evaluaciones externas. Cultura y Educación, 23(2), pp. 171-182. http://dx.doi. org/10.1174/113564011795944758 
Pérez, G. y Sarrate, M. L. (2013). Diversidad cultural y ciudadanía. Hacia una educación superior inclusiva. Educación XXI. 16(1), pp. 85-104. https://doi.org/10.5944/ educxx1.16.1.718

Pérez, G., Poza-Vilches, F. y Fernández-García, A. (2016). Criterios para una intervención de calidad con jóvenes en dificultad social. Revista Española de Pedagogía, 263, pp. 51-69.

Sánchez Pérez. J. (2016). Los riesgos psicosociales en el ámbito laboral: una visión global práctica. Granada: Comares.

Prats, E. (2010). Equidad y eficacia en la Estrategia de Lisboa: un reto estructural en los sistemas de educación básica en Europa. Revista Española de Educación Comparada, 16, pp. 131-158.

Pulido, R., Ballén, M. y Zúñiga, F.S. (2007). Abordaje hermenéutico de la investigación cualitativa. Teorías, Procesos, Técnicas. Colombia: Universidad Cooperativa de Colombia.

Represas, N. (2014). Política educativa de la UNESCO: reflexiones desde un modelo de análisis supranacional. Bordón, 67(2), pp. 101-115. http://doi.org/10.13042/ Bordon.2015.67207

Rodríguez, H., Ríos, O. y Racionero, S. (2012). Reconfiguración de la educación compensatoria en base a las evidencias científicas. Actuaciones inclusivas para la igualdad de resultados. Revista de Educación, n. ${ }^{\circ}$ extraordinario, pp. 67-87. http://doi.org/10.4438/1988-592X-RE-2012-EXT-207

Ron-Balsera, M., Dorsi, D., y Petersen, T. (2016). "Ensuring Mixed Education Systems Comply with Human Rights." A Report to The International Commission on Financing Global Education Opportunity. http://report.educationcommission. org/download/807

Sánchez, J. y Manzanares, A. (2014). Tendencias internacionales sobre equidad educativa desde la perspectiva del cambio educativo. Revista Electrónica de Investigación Educativa, 16(1), pp. 12-28.

Seibold, J. R. (2000). La calidad integral en educación. Reflexiones sobre un nuevo concepto de calidad educativa que integre valores y equidad educativa. Revista Iberoamericana de Educación, 23, pp. 215-231.

Serrano, I., Palomares, A. y Kostich, C. (2016). Metodologías que garantizan la inclusión educativa de estudiantes con discapacidad visual grave o ceguera. Cultura y Educación, 28(2), pp. 359-377. https://doi.org/10.1080/11356405.2016.1170336

Torres, J. (s.f.). 12 razones para decir NO a la LOMCE. Yo estudié en la pública. [Documento en línea] Disponible: https://www.yoestudieenlapublica.org/ descargas/16articulo12RazonesLeyLOMCE.pdf

Torres, J. A. y Castillo, S. (2016). Incidencia de las políticas de apoyo educativo en las estructuras organizativas de los centros desde la perspectiva del profesorado: un estudio en la provincia de Jaén. Educación XXI, 19(2), pp. 205-228. https://doi. org/10.5944/educxx1.16462 
Vélaz-de-Medrano, C. y Rodríguez, M. (2012). La incidencia de las políticas públicas en la equidad de la educación básica: estudio de casos múltiple en África subsahariana, Centroamérica y Magreb. Cultura y Educación, 24(1), pp. 45-60. https://doi. org/10.1174/113564012799740786

Vez, J. M. (2009). Educación Lingüística y cultural para una ciudadanía diversa y diferente. Cultura y Educación, 21(4), pp. 469-483. https://doi. org/10.1174/113564009790002418 